\title{
An optimised system for energy monitoring and data acquisition in substations/domestic applications using loT
}

\author{
V. Badri Rama Krishnan ${ }^{*}$, Keerthana Sandepudi ${ }^{1}$, and Shaista Gazal $^{1}$ \\ ${ }^{1}$ G.Narayanamma Institute Of Technology and Science, Electrical and Electronics Engineering Department, Hyderabad, Telangana, India
}

\begin{abstract}
The rising demand and tariff rates of electric power have derived the need to develop innovative methods to monitor, manage, economize and save energy. Energy monitoring and conservation are pre-eminent today because of the imbalance between power generation and demand. This paper proposes the outline and application of electrical energy measurement by an Arduino micro-controller that is used to calculate the power consumed by any individual electrical device. The main intention of the presented energy meter is to monitor, acquire and interpret the power consumption data online by integrating smart plugs, sensors at the device level and upload it to the server using IoT based communication protocol. Thus, this project enables the consumer to act accordingly to save power and to render a reliable power supply by making maximum use of energy sources in the cheapest ways.
\end{abstract}

\section{Introduction}

The data acquired by a system including software and hardware allows one to measure the energy consumed. Efficient usage of energy in smart homes reserves capital, sustainability and decreases carbon footprint at the whole. Necessarily, the need for smart energy management has ascended for the suburbans. However, the expense, complexity and high maintenance technology has somewhat limited a large-scale implementation of such systems. As IoT technologies are ubiquitous, they can provide a large scale computing platform for sensing, monitoring and controlling the household appliances energy consumption. This data can be collected using different wireless sensors which are installed in residential areas. Using predictive analysis and advanced methods, the data can be collected, analyzed and monitored in the form of reports, graphs and charts. Thus, this analyzed data can help common public, utilities, suppliers and utility operational stakeholders to gain significant intuitions on energy consumption of smart homes. The energy suppliers can use the power consumption data available to give an adaptable and on-demand supply with pertinent energy marketing plans [1]. Having a easy access to the electricity utilities and knowledge of their of consumption history, the users can adjust and optimize their power consumption and reduce their overall electricity bills. To have an functional cost saving system, it becomes necessary to monitor and control the operation of domestic applications depending on the collective power consumption over a time period, the maximum power consumption, the effect of weather/atmospheric conditions and consumption slab rates. This is where the combination of IoT technology, energy management solutions on a local and national level can be implemented using Big Data analytics and BI [2]. Moreover, the use of IoT also allows seamless remote control of home electronics permitting the customers to their ON/OFF usage pattern via a personal computer or a mobile phone.
The monitoring of power consumption and energy of residential loads using ZigBee as the communication protocol was first introduced. Although, the data that was collected and aggregated solely by the home server in this system could lead to data loss in case of a system shut-down. Additionally, a bridge between ZigBee and TCP/IP stack would be needed to connect it to a community of homes. It has also been extended to extensive domains in the IoT paradigm employing the GSM/GPRS networks to remotely control the end-devices [3]. Correspondingly, authors in [4] emphasize on IoT based DC powered homes to develop a DC distribution system circumscribing all residential DC-based loads that interact with each other. Nevertheless, the dearth of standardized protocols and regulations were the main challenges in considering smart DC powered homes as appropriate substitute to AC power systems. IoT provides an coherent interface for DC powered technologies in economic energy distribution to combat these problems.

Installing home EMS can help the public-owners to comprehend contribution of each device towards the electricity bill they receive every month. Furthermore, most of former work has basically prioritised individual smart homes and lack the energy management provisions for regional utility providers or national level utility centers. The technology to collect huge volumes of data from home sensor networks is available, however, managing the collected data efficiently and extracting deeper insights from it remains a challenge.

The existing models on EMS and cost saving models are implemented on discrete units while the proposed model can be built on top of the existing paradigms to provide a distributed EMS platform from consumer to community levels. It has allies found from a study found that home energy management system can reduces electricity consumption by $17-20 \%$ potentially with tolerable discomfort [5].

Smart energy meter[6] is developed using the Wifi module based on three main objectives. They are:- 
1. To provide automated load energy immediately.

2. To optimize the usage of electricity.

3. To reduce power wastage and make it more economic.

The proposed system design removes human inclusion in electricity maintenance and introduces cost-effectiveness. It allows the consumer to audit the energy consumption in watts from a webpage. The webpage evaluates and provides a detailed description and visualization of the energy usage statistics. Wi-Fi unit performs IoT operation by transferring the data of the load to Google sheets(docs). Hence, the consumer can manage power by realizing the energy usage time to time. This system utilizes an Arduino microcontroller. The units that are generated are displayed via the Wifi module. The IoT hypothesis gives the basic framework and opportunities to form a connection between the physical world and computer based systems. Hardware devices are connected with each other over the internet.

\section{Components Required}

1. SENSOR DATA: Smart plugs (Sensors MODULES)

\section{GATEWAY (WIFI Modules)}

3. GOOGLE SHEETS (that will help individual users to post and retrieve sensor data)

\subsection{Block diagram}

The smart electricity meter consists of an ARDUINO Uno Board, ESP8266 WiFi module, current and voltage sensors and a power supply.

The system is designed on an ARDUINO microcontroller. It's structure is differentiated into three sections viz., the controller, sensors and a WiFi unit. The controller does the basic calculations and processes the information. The voltage and the currents sensors transfer the readings to ESP8266 with the help of the CP2102 USB to UART driver and upload to Google sheets using the Wifi module.The system sample the signals and converts the resulting samples into digital numeric values that can be manipulated by a computer. The components of data acquisition systems include:

- Sensors that convert the physical parameters to electrical signals.

- Signal conditioning circuitry that converts the sensor signals to be further converted into digital values.

- Analog-to- digital converters that convert conditioned sensor signals to digital values.

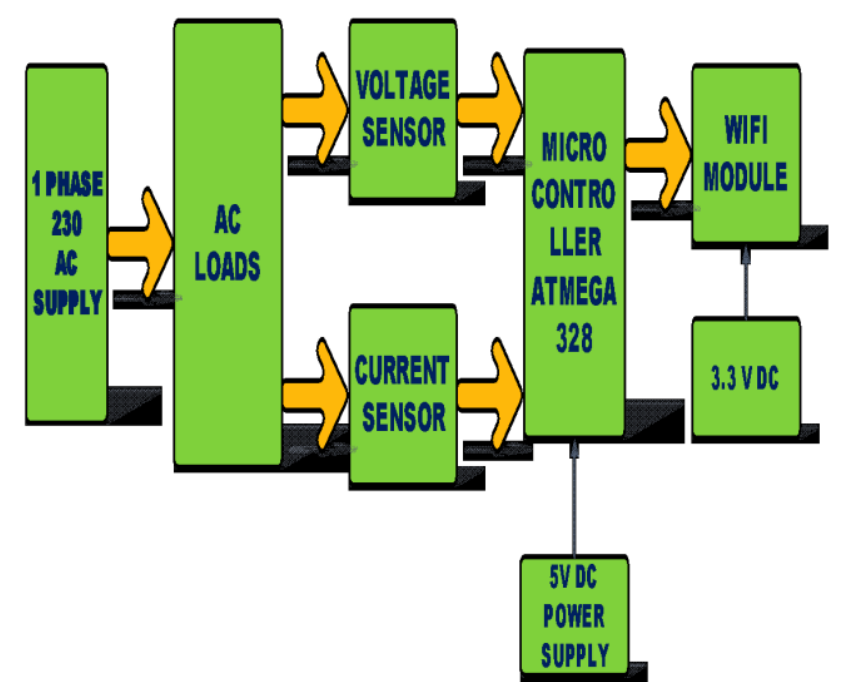

Fig. 1. Block diagram of the electricity meter

\section{Hardware Implementations}

\subsection{Current Sensing}

The measurement of AC current has always been laborious due to coupling of noise signals with its inappropriate isolation etc. But, with the help of ACS712 module devised by Allegro Microsystems, it has become a lot easier. According to the Hall-effect, when a current carrying conductor is placed in a magnetic field, an emf is generated across its edges perpendicular to the directions of both the current and the magnetic field that is measured by the hall sensor. This is called as the hall-voltage, measured in terms of millivolts. The measured hall-voltage is directly proportional to the current flowing through the conductor.

The major advantage of using ACS712 Current Sensor is that it can measure both $\mathrm{AC}$ and $\mathrm{DC}$ currents and it also provides isolation between the Load (AC/DC load) and the Measuring Unit (Microcontroller part). As shown in the picture we have three pins on the module which are Vcc, Vout and Ground respectively. The current from output voltage of ACS712 current sensor can be calculated as follows:

- When there is no current flowing through the sensor, output voltage will be Vcc / 2. Where Vcc is power supply voltage given to acs 712 to current sensor.

- If the $\mathrm{Vcc}=5$ volt, then the output voltage of current sensor will be equal to 2.5 when there is no current passing through a sensor. 2.5 volt is the offset voltage or base voltage of the sensor which should be subtracted from the measured voltage.

- The output voltage decreases when current starts passing through the sensor.

- So we can calculate dc current by using following commands: 
adcvalue $=$ analogRead $(\mathrm{A} 0)$

Voltage $=($ adcvalue $/ 1024.0) * 5000$;

Current $=(($ Voltage- voltage_offset $) / \mathrm{mV}$ perAmp $)$;

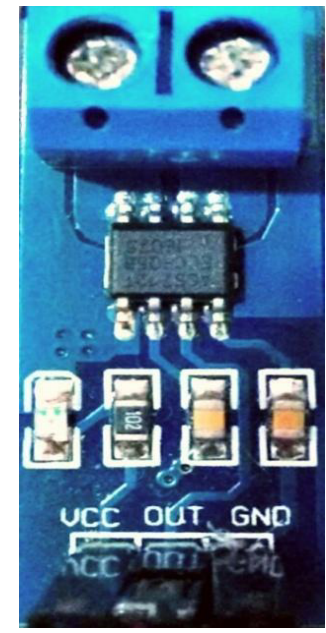

Fig. 2. ACS712 Current Transformer

\subsection{Voltage Sensing}

ARDUINO is generally operated and manufactured to work with voltage ratings less than or equal to $5 \mathrm{~V}$. So, it is not possible to directly measure AC voltage greater than $230 \mathrm{~V}$ by giving high input voltages to microcontrollers which may cause temporary or permanent damage to microcontrollers. Hence, a simple transformer with less current rating which steps down $230 \mathrm{~V}$ to $12 \mathrm{~V}$ is used. The voltage divider decreases the voltage being measured from $12 \mathrm{~V}$ to $5 \mathrm{~V}$. Thereby, ARDUINO analog inputs can be used to measure DC voltage between 0 and $5 \mathrm{~V}$ (on $5 \mathrm{~V}$ ARDUINOS such as the Arduino Uno when using the standard 5V analog reference voltage). Code in the ARDUINO sketch is then used to calculate the actual voltage being measured. This allows voltages greater than $5 \mathrm{~V}$ to be measured.

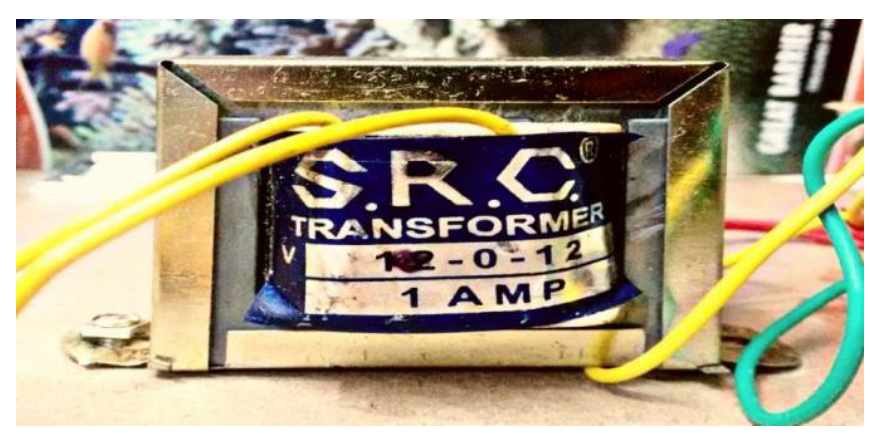

Fig. 3. Potential Transformer

The input voltage value is given to pin A2 of the Arduino. It provides some simple filtering by adding up 10 analog values from pin A2 sampled at $10 \mathrm{~ms}$ intervals. After taking
10 samples, the voltage is calculated using the average of the 10 sampled values and is sent out of the serial port for display on the Arduino Serial Monitor window. Ten analog samples are taken using the following code 1 . The sum or total of all 10 values added together are stored in sum. The variable sample count keeps track of the number of samples. The voltage is calculated using:

voltage $=(($ float $)$ sum $/($ float $)$ NUM_SAMPLES $* 5.0) /$
$1024.0 ;$

A calibrated value is used in place of 5.0 in the above sketch. The 10 samples (sum) is divided by 10 (NUM SAMPLES) to get the average value read.5.0 is the 5V ADC reference voltage. 1024.0 is the maximum value that the ADC can have plus $1(1023+1$ or 2 to the power of 10 plus 1) 1023.0 can also be used here.

\section{Operation}

The supply voltage is connected to the AC loads with the current sensor in series and the transformer connected in parallel. The sensed values of current and voltage are given to the $\mathrm{A} 1$ and $\mathrm{A} 2$ pins of the Arduino respectively. This ARDUINO is based on ATmega328 microcontroller where the program to evaluate the sensed values is loaded into. Now, the IoT operation is performed by ESP8266 Wifi module by sending energy meter data to web-page which can be accessed through an IP address. The Tx, Rx of ESP8266 pins are connected to the pins $7 \& 8$ of the ARDUINO board. CP2102 is an USB2.0 to TTL UART Converter module based on CP2102 Bridge by SiLabs. It is used with Laptop's not having a standard serial port. This module creates a virtual COM port using USB on your computer which can support various standard Baud Rates for serial communication. The $\mathrm{Rx}$ and Tx pin can be connected directly to the MCUs pins (assuming $5 \mathrm{v} \mathrm{I} / \mathrm{O}$ ). The suitable interfacing between Arduino with ACS712 and arduino with ESP8266 is done and the respective codes are dumped on to it using Arduino computer program interface.

When the supply is given and the Arduino code is executed, the sensed voltage and the current readings are transferred to ESP8266 with the help of the CP2102 USB to UART driver. By following the TCP/IP stack protocol, we send this data to the suitable webpage using a WIFI router. By using Google sheets, we list down the values of voltage, current and power being updated continuously over the required period of time. Using ESP8266 User Data Program (UDP) protocol data can be sent to local routers or intermediate stations for data continuity or redundancy. Hence provides extra support for data transfer during internet shutdowns too. Analysis of power quality will be much more user or customer understandable with the Google sheets data platform, As the data is free to be utilized with many simulation as well as real time data software's. 


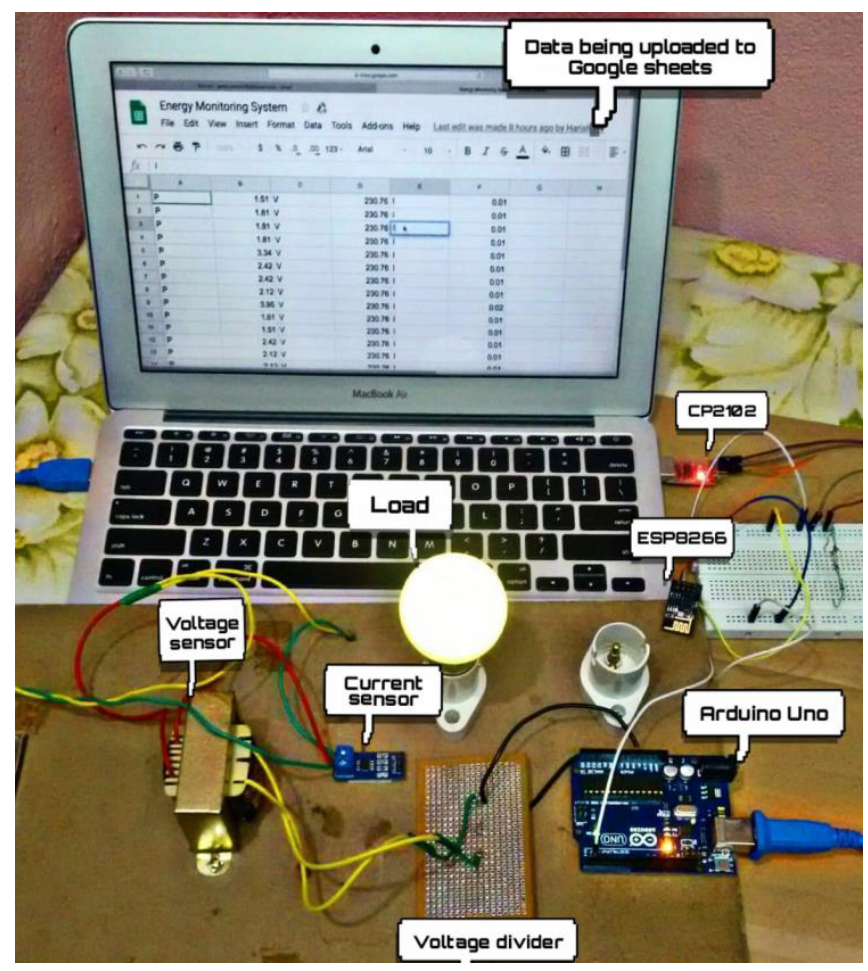

Fig. 4. Hardware Circuitry with output being displayed in the Google sheets

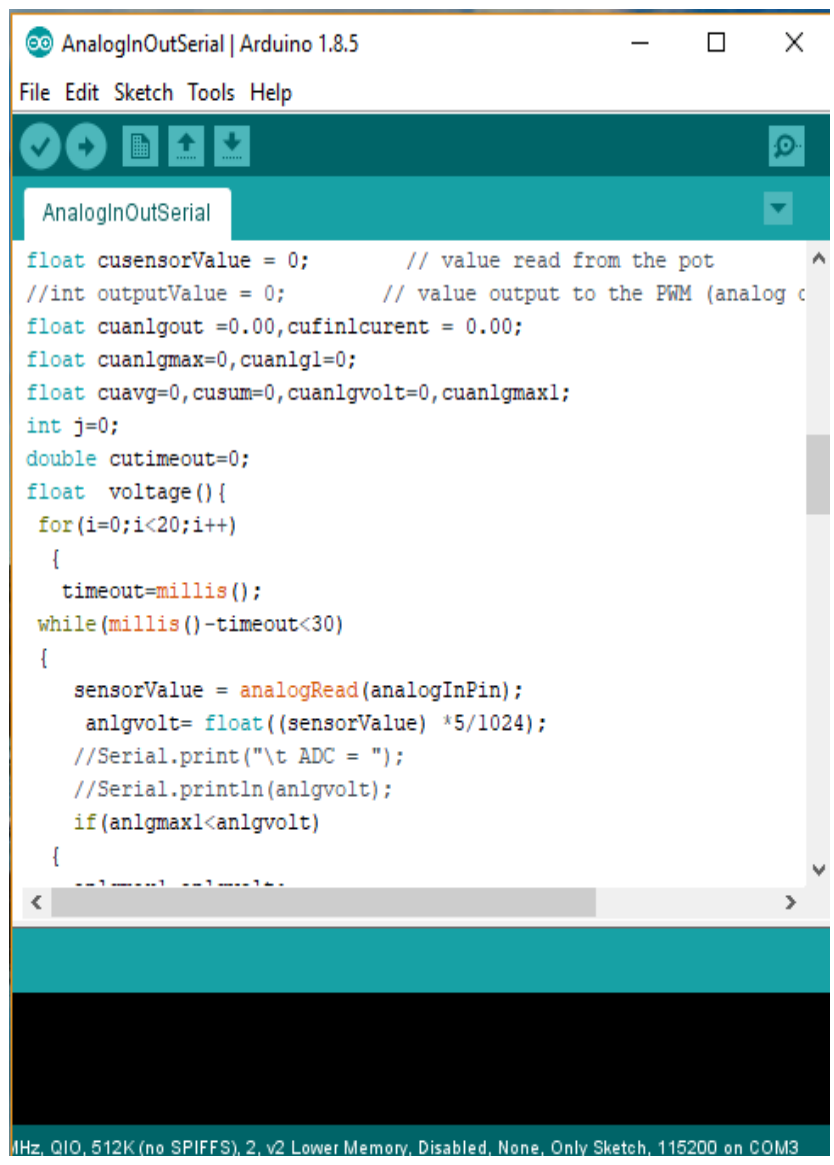

Fig. 5. Code for Arduino uno
(2) udprecev | Arduino 1.8.5

File Edit Sketch Tools Help

$\checkmark$ ( $)$ 通 \pm

udprecev

Finclude 〈ESP8266WiFi.h〉

\#include 〈WiFiUdp.h>

\#include 〈WiFiclientSecure. $\mathrm{h}$ >

const char* ssid = "keerthana";

const char* password = "keerthana $26 "$.

const chark host $=$ "script.google.com";

const int httpsPort $=443$;

WiFiUDP Udp;

unsigned int localUdpPort $=4210$; / local port to listen on

char incomingPacket [255]; // buffer for incoming packets

char replyPacket[] = "Hi there! Got the message :-)"; // a repls

int rec, incomingByte $=0$;

const int bSize $=30$;

char Buffer[bSize]; // Serial buffer

char Command10]; // Arbitrary Value for command size

char Data[15]; // ditto for data size

int ByteCount;

String buf;

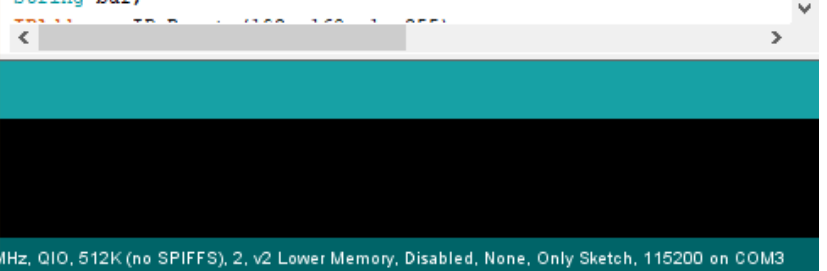

Fig. 6. Code for ESP8266 Wifi Module

\section{Results}

The power, voltage and current values sensed and measured are seen in the below picture in the serial monitor of the Arduino. The data Is further sent to the ESP8266. The measured data from the ESP8266 is transmitted to the Google sheets following the TCP/IP protocol as shown in fig. 7. and fig. 8 .

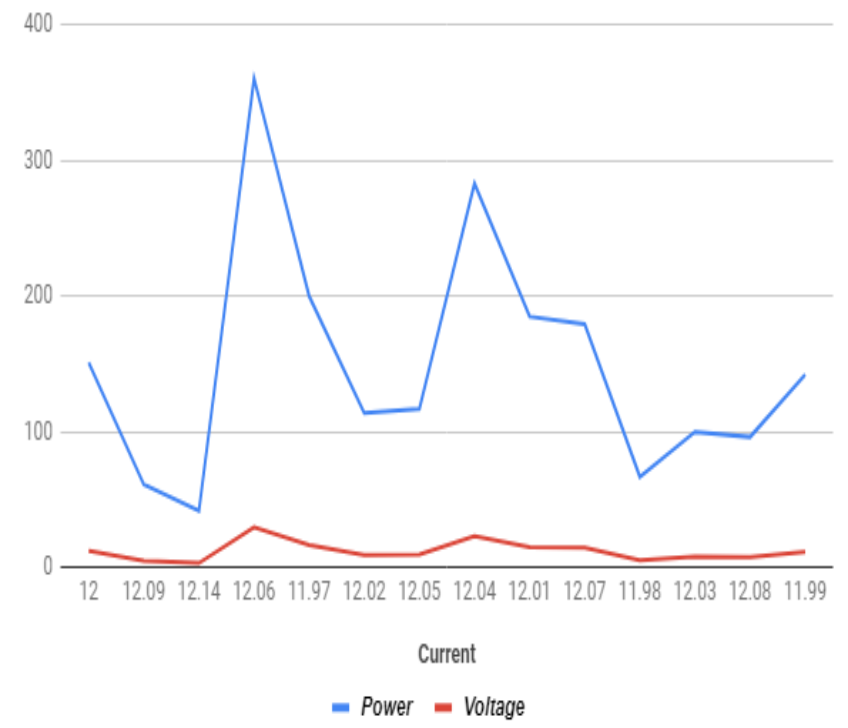

Fig. 7. Output power, voltage and current as displayed in Google sheets chart 


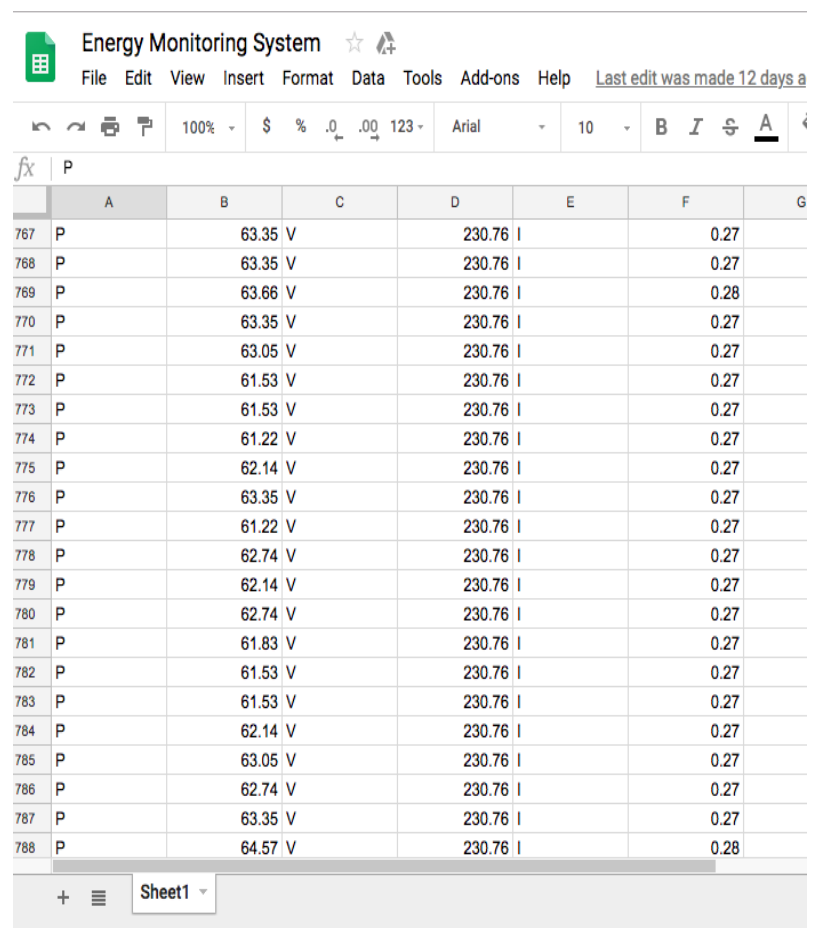

Fig. 8. Output power, voltage and current as displayed in Google sheets

\section{Conclusion}

The proposed design is set to unfurl a new approach for smart energy management on the IoT platform. The system allows its users to monitor and control devices remotely via a userfriendly interface. The google sheets data provides a platform for many other vendors to use this data for further improvement of the power quality of the system. Data obtained can be sent to other cloud applications or Artificial Intelligence techniques can be enforced to predict further data analysis.

Tracking the performance of your electrical devices and system is also possible by power and energy management system. It provides real-time monitoring of the electrical parameters and makes you aware of any irregularities. That helps you to recognize and address any potential problems before the power supply completely goes off. The system also gives you data on how well your electrical system is supplying power to the devices and critical loads, helping to fortify optimal efficiency and safety for the electrical system and its various components.

\section{References}

1. International Research Journal of Engineering and Technology (IRJET), "IoT Based Smart Energy Monitoring”, e-ISSN: 2395-0056 Volume: 05 Issue: 03 | Mar-2018.

2. IEEE Transactions on Consumer Electronics, "A Smart Home Energy Management System Using IoT and Big Data Analytics Approach", Vol. 63, No. 4 | November 2017.
3. G. Mingming, S. Liangshan, H. Xiaowei and S. Qingwei, "The System of Wireless Smart House Based on GSM and ZigBee", 2010 International Conference on Intelligent Computation Technology and Automation, Changsha $\mid$ 2010, pp. 1017-1020.

4. E. Rodriguez-Diaz, J. C. Vasquez and J. M. Guerrero, "Intelligent DC Homes in Future Sustainable Energy Systems: When Efficiency and Intelligence Works Together", IEEE Consumer Electron. Magazine, vol. 5, no. 1, pp. 74-80 | Jan. 2016.

5. K.Dittawit and F. A. Aagesen, "Home energy management system for electricity cost savings and comfort preservation", 2014 IEEE Fourth International Conference on Consumer Electronics Berlin (ICCEBerlin), Berlin, 2014, pp. 309-313.

6. Internet of Things (Iot) Based online Energy Monitoring System, Prof.M.Chakravarthy.

7. http://microcontrollerslab.com/acs712-current-sensorinterfacing-arduino/.

8. http://microcontrollerslab.com/data-receivingwebpage-arduino-esp8266/.

9. http://engineerexperiences.com/acvoltagemeasurement.html.

10. https://www.silabs.com/products/interface/usbbridges/classic-usb-bridges/device.cp2102. 